\title{
Defining gastrointestinal transit time using video capsule endoscopy: a study of healthy subjects
}

\section{다)(1) $\odot$}

Authors

John O’Grady ${ }^{1,2}$, Clodagh L. Murphy',2, Lillian Barry³, Fergus Shanahan ${ }^{1,2}$, Martin Buckley ${ }^{3}$

Institutions

1 APC Microbiome Ireland, University College Cork, National University of Ireland, Cork, Ireland

2 Department of Medicine, Cork University Hospital, National University of Ireland, Cork, Ireland

3 Centre for Gastroenterology, Mercy University Hospital, Cork, Ireland

submitted 23.7.2019

accepted after revision 11.11 .2019

\author{
Bibliography \\ DOI https://doi.org/10.1055/a-1073-7653 | \\ Endoscopy International Open 2020; 08: E396-E400 \\ (c) Georg Thieme Verlag KG Stuttgart · New York \\ eISSN 2196-9736
}

Corresponding author

Dr. John O'Grady, Department of Medicine, Floor 1 Clinical Sciences Building, Cork University Hospital, Cork, Ireland Fax: +0214922000

john.ogrady@ucc.ie

\section{ABSTRACT}

Background and study aims Determining the etiology and location of gastrointestinal motility disorders can be challenging. A range of investigations targeting specific areas of gastrointestinal transit are available, but many provide clinical data for a given gastrointestinal region alone or for non-specific whole gut transit, and are otherwise of limited use. Video capsule endoscopy allows endoscopic visualisation of the entire gastrointestinal tract, and may also provide more specific data for regional transit time abnormalities.

Patients and methods Data from video capsules ingested by 71 ambulatory healthy subjects were recorded and analyzed to determine gastric and small bowel transit times in the fasting state.

Results Median, and interquartile range (IQR), gastric transit time was $22(10-48)$ minutes, and median (IQR) small bowel transit time was 198.5 (157-240.5) minutes.

Conclusion These data, for the first time to our knowledge, provide references for gastrointestinal transit times among healthy ambulatory subjects using video capsule endoscopy. This potentially strengthens clinical use of video capsule endoscopy in the investigation of patients with suspected gastrointestinal motility disorders.

\section{Introduction}

Diagnosis of gastrointestinal motility disorders is challenging partially due to a lack of attainable and useful data from less accessible regions of the gut such as the small intestine [1]. Image-based assessment of intestinal pathology such as x-ray, computed tomography (CT), magnetic resonance imaging (MRI) and direct endoscopic visualisation are frequently relied on to formulate a diagnosis. Current investigations for intestinal motility abnormalities include antro-duodenal manometry, use of radiopaque markers, scintigraphy, hydrogen breath testing as well as newer techniques such as video capsule endoscopy, wireless motility capsule, and MRI- based motility assessment $[1,2]$. Scintigraphy is the current gold standard for measurement of gastric emptying and small bowel transit time [2]. However, motility related symptoms are non-specific and often correlate poorly with results of transit time analyses [3].
Many of these methods are time-consuming, expensive, invasive, and some, such as scintigraphy, may involve significant levels of radiation exposure depending on the radioactive meal and clinical indication [2,4]. Furthermore, there are inter- investigational differences as to which anatomical area of the gut data are obtained from. For example, antro-duodenal manometry record data for specific segments of the intestine, while radiopaque markers and hydrogen breath testing measures whole gut or oro-cecal transit. The wireless motility capsule, by comparison, estimates and records intestinal transit times, following a meal, by measuring changes in $\mathrm{pH}$, temperature and pressure $[5,6]$. In addition, both individual variability (of up to $50 \%$ [2]) and lack of agreement between investigations on transit times prevents definition of a universal reference range [7].

Few investigations allow both direct imaging of intestinal structures as well as calculation of transit time. Video capsule 


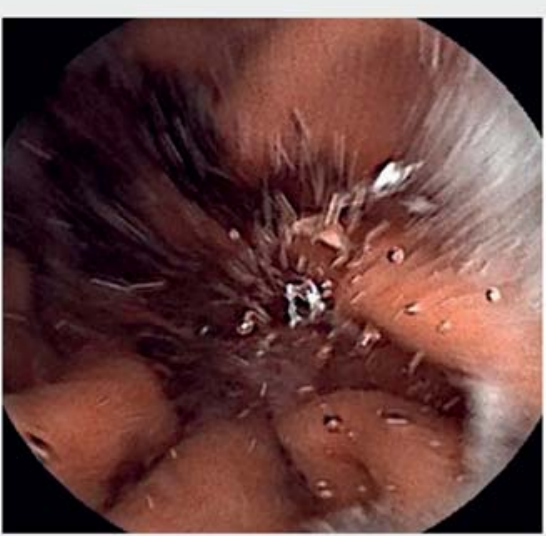

First Gastric Image

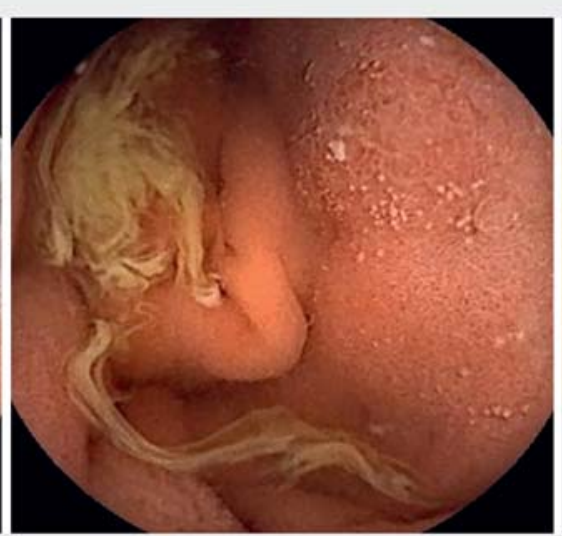

First Duodenal Image

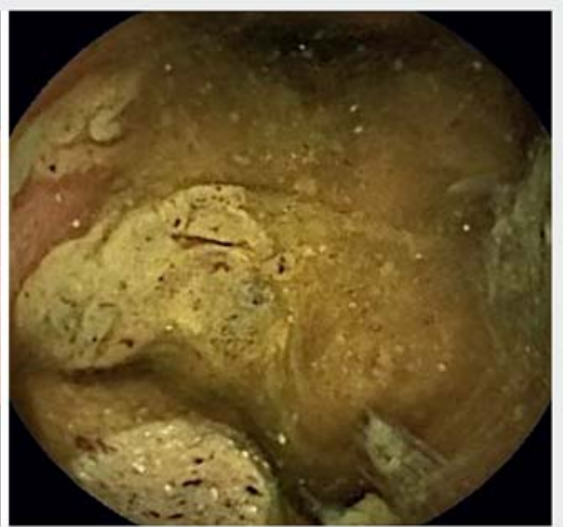

First Caecal Image

Fig. 1 Examples of first gastric, duodenal and caecal images recorded using video capsule endoscopy. These images identify landmarks that allow gastric and small intestinal transit time calculation.

endoscopy is one such modality that incorporates luminal visualisation, similar to standard endoscopy, as well as providing transit time data for the stomach and small intestine $[7,8]$. Transit time can be calculated through the identification of anatomical landmarks [7]. Video capsule endoscopy may, therefore, have a role in evaluating symptoms suggestive of gastrointestinal motility disorders as well as other intestinal pathology. However, as video capsule endoscopy requires ingestion of a capsule, a suspected stricture contraindicates its use, at least initially [2]. Video capsule endoscopy is also performed in the fasting state as opposed to the fed state required for studies such as the motility capsule. A further current limitation of video capsule endoscopy as a means of investigating motility disorders is the lack of normal values and clinical significance of transit times which hitherto has been unclear. To date, many of the trials of video capsule endoscopy have involved patients with varying medical conditions such as unexplained iron deficiency anaemia [7], occult gastrointestinal blood loss [9, 10], celiac disease [11], functional bowel disorders [12], and critically ill patients [13]. This study aimed to assess gastric and small bowel transit times for healthy ambulatory male and female subjects to establish norms that can inform future diagnostic value of video capsule endoscopy.

\section{Patients and methods}

Healthy male and female subjects without pre-existing gastrointestinal symptoms or conditions were included. Exclusion criteria included smokers and pregnant subjects, as well as those with a history of bleeding disorders, inflammatory bowel disease (IBD), irritable bowel syndrome (IBS), previous intestinal surgery, diabetes, and use of medications that affect gastrointestinal motility.

The trial was conducted in accordance with the ethical principles set forth in the current version of the Declaration of Helsinki, the International Conference on Harmonization E6 Good Clinical Practice (ICH-GCP). Ethical approval was granted by
The Clinical Research Ethics Committee of the Cork Teaching Hospitals (Cork, Ireland), and informed consent was sought from each subject. The trial was conducted from July to December 2017. The trial was registered at ClinicalTrials.gov under the ID number NCT03228589. This study was performed as a screening of healthy subjects for involvement in this clinical trial.

Capsule endoscopy was performed using the SB3 Pillcam video recording capsule (Medtronic, Ireland). Subjects were fasted from midnight the night before. In the morning, the capsule was swallowed with water and subjects then continued fasting for a further 4 hours. While allowed to leave and carry out usual activities of daily living, the subjects did not engage in strenuous exercise during the 8 -hour recording period. The Pillcam receiver belt was returned after this time and video images were retrieved for analysis.

Gastric transit time (GTT) is defined as time from first gastric image to first duodenal image, and small bowel transit time (SBTT) is defined as time from first duodenal image to first cecal image ( $>$ Fig.1). All video capsule endoscopies were reviewed by two expert gastroenterologists with experience in interpretation and in cases of discrepancy, a third clinician reviewed the data.

Data were analyzed using Microsoft Office Excel and IBM SPSS Version 24.0 (IBM Corp 2016). Data are reported as mean \pm standard deviation (SD) for evenly distributed data, and median \pm inter-quartile range (IQR) for non-evenly distributed data.

\section{Results}

A total of 71 video capsule endoscopy studies performed, in which first gastric, duodenal and caecal images were identified, were suitable for analysis. The age range was $19-40$ years. The group mean age, and SD, was $30.49 \pm 6.7$ years. $40(56.3 \%)$ of the study participants were female, and mean body mass index (BMI), and SD, was $24.25 \pm 2.16$. All video capsule images were 
- Table 1 Sample comparison of gastric and small bowel transit times from studies using video capsule endoscopy fasting protocol [5, 7,9-12,16-18]

\begin{tabular}{|c|c|c|c|c|c|}
\hline Study & Subject & GTT (minutes) & SBTT (minutes) & Capsule Endoscopy Indication & $\%$ in colon \\
\hline Current data & $\mathrm{n}=71$ & $\begin{array}{l}\text { Median (IQR): } \\
22(10-48)\end{array}$ & $\begin{array}{l}\text { Median (IQR) } \\
198.5(157-240.5)\end{array}$ & Healthy control & Incomplete eliminated \\
\hline Prichard et al & $n=59$ & $\begin{array}{l}\text { Median (IQR): } \\
25(14-55)\end{array}$ & $\begin{array}{l}\text { Median (IQR): } \\
222(151-287)\end{array}$ & $\begin{array}{l}\text { Gastrointestinal bleed/ } \\
\text { diarrhea/IBD }\end{array}$ & $97 \%$ \\
\hline Hejazi et al & $n=139$ & $\begin{array}{l}\text { Median (IQR): } \\
20(12-36)\end{array}$ & $\begin{array}{l}\text { Median (IQR): } \\
216(150-294)\end{array}$ & IDA/gastrointestinal bleed & $82.3 \%$ \\
\hline $\begin{array}{l}\text { Diaz Tartera } \\
\text { et al }\end{array}$ & $n=70$ & $\begin{array}{l}\text { Median: } \\
26\end{array}$ & $\begin{array}{l}\text { Median: } \\
236\end{array}$ & $\mathrm{IDA} / \mathrm{FOB}+$ & Incomplete eliminated \\
\hline Ou et al & $n=62$ & $\begin{array}{l}\text { Median: } \\
19.43\end{array}$ & $\begin{array}{l}\text { Median } \\
232.52\end{array}$ & IBD/gastrointestinal bleed & $88.71 \%$ \\
\hline Pioche et al & $\begin{array}{l}n=73 \\
\text { (2 VCE systems) }\end{array}$ & $\begin{array}{l}\text { Mean (range): } \\
\text { Pillcam SB2 system: } \\
37.8 \text { (16-203) } \\
\text { Mirocam system: } \\
47.9(3-232)\end{array}$ & $\begin{array}{l}\text { Mean (range): } \\
\text { Pillcam SB2 system: } \\
234.5 \text { (51-502) } \\
\text { Mirocam system: } \\
268.1 \text { (58-538) }\end{array}$ & Unexplained blood loss & $77 \%$ \\
\hline $\begin{array}{l}\text { Velayos Jimé- } \\
\text { nez et al }\end{array}$ & $\mathrm{n}=89$ & $\begin{array}{l}\text { Mean (range): } \\
22.6(0.7 \text { to } 171)\end{array}$ & $\begin{array}{l}\text { Mean (range): } \\
283.3 \text { (91 to } 416 \text { ) }\end{array}$ & Undefined & Retrospective \\
\hline $\begin{array}{l}\text { Malagelada } \\
\text { et al }\end{array}$ & $\mathrm{n}=70$ & $\begin{array}{l}\text { Mean }( \pm \text { SE): } \\
41( \pm 5)\end{array}$ & $\begin{array}{l}\text { Mean }( \pm \mathrm{SE}) \text { : } \\
218 \pm 12\end{array}$ & $\begin{array}{l}\text { Healthy controls in comparison } \\
\text { to functional bowel symptoms }\end{array}$ & $74 \%$ \\
\hline Urgesi et al & $\begin{array}{l}\mathrm{n}=30(\text { coeliac }) \\
\mathrm{n}=30(\text { control })\end{array}$ & $\begin{array}{l}\text { Mean }( \pm S D) \text { : } \\
\text { celiac disease: } \\
30.4 \pm 21.9 \\
\text { Control: } \\
43.6 \pm 48.8\end{array}$ & $\begin{array}{l}\text { Mean }( \pm \text { SD): } \\
\text { celiac disease: } \\
252.2 \pm 67.4 \\
\text { Control: } \\
244.7 \pm 88.4\end{array}$ & $\begin{array}{l}\text { Celiac disease/gastrointestinal } \\
\text { bleed/IDA }\end{array}$ & Retrospective \\
\hline Fireman et al & $\mathrm{n}=40$ & $\begin{array}{l}\text { Mean: } \\
45.5\end{array}$ & $\begin{array}{l}\text { Mean: } \\
218\end{array}$ & IDA/pain/IBD & $82.1 \%$ \\
\hline
\end{tabular}

within normal limits. This was expected as these video capsule studies were performed as screening of healthy subjects for involvement in clinical trial NCT03228589.

Median (and IQR) gastric transit time was 22 minutes (range 10-48), and median (IQR) small bowel transit time was 198.5 minutes (range 157-240.5). A wide range was observed for both gastric (1-179.5 minutes) and small bowel (41.5-422 minutes) transit times. For the female participants, the median gastric and small bowel transit times, respectively, were 27.5 minutes (IQR 12.4-46.3) and 189.2 minutes (IQR 144.25232.25) minutes. For males, median gastric and small bowel transit times, respectively, were 20 minutes (IQR 8.5-50) and 210 minutes (IQR 165.5-246). The Differences between genders were not found to be statistically significant for either gastric or small bowel transit, $P=0.754$ (gastric) and $P=0.195$ (small bowel).

\section{Discussion}

Using video capsule endoscopy, in healthy ambulatory subjects a median gastric transit time of 22 (IQR 10-48) minutes was observed in the fasting state. There was no significant difference observed for gender or BMI, which correlates with pre- vious studies using other investigative techniques [14] and studies involving video capsule endoscopy [15]. By means of comparison, - Table 1 summarizes gastric transit times outlined in other video capsule endoscopy studies [5, 7, 9-12, 1618]. Importantly, only one study was completed using healthy controls [12]. However, these patients remained lying down for 8 hours after capsule ingestion and as opposed to undertaking usual activities of daily living which would be standard protocol for capsule endoscopy. The remainder of the studies were completed on patients for investigation of medical conditions including IBD and gastrointestinal bleeding [9] and iron deficiency anemia [7] and therefore may not be representative of a "healthy population" for defining a normal reference range. The broad range observed in our data for gastric transit time may be, in part, due to different mechanisms of non-digestible solid emptying, such as phase III migrating motor complex (MMC) and isolated antral contractions [19].

Our video capsule endoscopy data report median small bowel transit time (IQR) of 198.5 (157-240.5) minutes. Again, no significant differences were observed for gender or BMI. $>$ Table 1 summarizes data on small bowel transit times from previous studies for comparison. As with gastric transit studies, much of the available data for small intestinal transit time using 
video capsule endoscopy is derived from studies of patients who underwent video capsule endoscopy for another clinical indication. These include investigation of iron deficiency anaemia, occult blood loss [7], celiac disease [11] and comparison of bowel preparations $[16,20]$. Similar to gastric transit time, the same single study evaluated small bowel transit times in healthy controls who were lying on a hospital bed for a period of 8 hours [12].

Gastric transit time from our data is similar to that of previous studies, while a possible shorter small bowel transit times is observed. This may be due to subjects in our study being ambulatory for the duration of the recording, or having no comorbid medical conditions or clinical indications for capsule endoscopy. All video capsule endoscopies were performed in the fasting state, as is standard protocol for video capsule endoscopy, and possibly explains shorter gastric transit compared with fed-state studies such as wireless motility capsule or scintigraphy.

Using our data, video capsule endoscopy may represent an alternative method of measuring gastrointestinal transit in the fasting state, as compared with fed-state techniques such as scintigraphy and breath testing, and also offers dual benefit of direct luminal visualization. Scintigraphy remains, however, the reference gold standard for fed-state measurement of gastric emptying and small bowel transit time [2]. A radio-labelled meal is consumed [21] with imaging taken at incremental stages to estimate transit times against a background reference range $[22,23]$. Delayed gastric emptying, for example, may be defined as retention $>90 \%$ at 1 hour, $>60 \%$ at 2 hours, and $>10 \%$ at 4 hours [24]. Radiation exposure is a significant limitation of the technique [4].

13C-gastric emptying breath testing may be used as an alternative to scintigraphy $[2,14]$. 13C-labelled substrate disintegrates in the duodenum to release 13-CO2 which can be analyzed and quantified by mass spectrometry [25]. The delay between ingestion of substrate and appearance of $\mathrm{CO} 2$ is indicative of gastric emptying time [25]. Unlike scintigraphy, this test is unable to assess regional transit abnormalities, instead giving a global overview of gastric emptying and it is also limited by subject baseline metabolic function and absorption [4]. Other investigations, such as wireless motility capsule, also limit radiation exposure and provide intestinal segment transit data but are performed in the fed-state and do not transmit endoscopic images.

Video capsule endoscopy as a technique that is free from radiation, which does not rely on metabolic parameters, that provides endoscopic images for analysis, and is performed in the fasting state, provides a unique dataset in the investigation of gastrointestinal disease.

\section{Conclusion}

This is, to our knowledge, the first report of gastric and small bowel transit times using video capsule endoscopy in healthy ambulatory subjects and may provide future reference for gastrointestinal motility disorder investigation. In future, video capsule endoscopy recordings using our data may assist in identifying patients with possible motility disorders who otherwise have normal mucosal findings. Such patients may then benefit from formal fed-state studies such as scintigraphy. Head-to-head study of the gold standard techniques such as Scintigraphy and video capsule endoscopy would be useful to further validate this data.

\section{Acknowledgements}

The trial was sponsored by Chr Hansen and run by Atlantia Food Clinical Trials.

\section{Competing interests}

Dr. Shanahan, Dr. O'Grady, and Dr. Murphy are funded, in part, by Science Foundation Ireland (APC/SFI/12/RC/2273) in the form of a research center, APC Microbiome Ireland. Dr. Shanahan is a cofounder, shareholder in Atlantia Food Clinical Trials, 4D Pharma Cork, Alimentary Health. He is the director of APC Microbiome Ireland, a research center funded in part by Science Foundation Ireland (APC/SFI/12/RC/2273) and which has recently been in receipt of research grants from AbbVie, Alimentary Health, Cremo, Danone, Janssen, Friesland- Campina, General Mills, Kerry, Mead Johnson, Nutricia, 4D Pharma and Second Genome, and Sigmoid Pharma.

\section{References}

[1] Gronlund D, Poulsen JL, Sandberg TH et al. Established and emerging methods for assessment of small and large intestinal motility. Neurogastroenterology and motility : the official journal of the European Gastrointestinal Motility Society 2017; 29: doi:10.1111/nmo.13008

[2] Keller J, Bassotti G, Clarke J et al. Expert consensus document: Advances in the diagnosis and classification of gastric and intestinal motility disorders. Nat Rev Gastroenterol Hepatol 2018; 15: 291-308

[3] Balan K, Sonoda LI, Seshadri N et al. Clinical significance of scintigraphic rapid gastric emptying. Nucl Med Commun 2011; 32: 11851189

[4] Knight LC. Update on gastrointestinal radiopharmaceuticals and dosimetry estimates. Semin Nucl Med 2012; 42: 138-144

[5] Diaz Tartera HO, Webb DL, Al-Saffar AK et al. Validation of SmartPill. Neurogastroenterol Motil 2017; 29: 1-9

[6] Farmer AD, Scott SM, Hobson AR. Gastrointestinal motility revisited: The wireless motility capsule. United European Gastroenterol J 2013; 1: 413-421

[7] Hejazi RA, Bashashati M, Saadi M et al. Video capsule endoscopy: a tool for the assessment of small bowel transit time. Frontiers Med 2016; 3: 6

[8] Pennazio M, Spada C, Eliakim R et al. Small-bowel capsule endoscopy and device-assisted enteroscopy for diagnosis and treatment of small-bowel disorders: European Society of Gastrointestinal Endoscopy (ESGE) Clinical Guideline. Endoscopy 2015; 47: 352-376

[9] Ou G, Svarta S, Chan C et al. The effect of chewing gum on smallbowel transit time in capsule endoscopy: a prospective, randomized trial. Gastrointest Endosc 2014; 79: 630-636

[10] Pioche M, Gaudin JL, Filoche B et al. Prospective, randomized comparison of two small-bowel capsule endoscopy systems in patients with obscure GI bleeding. Gastrointest Endosc 2011; 73: 1181-1188 
[11] Urgesi R, Cianci R, Bizzotto A et al. Evaluation of gastric and small bowel transit times in coeliac disease with the small bowel PillCam(R): a single centre study in a non gluten-free diet adult Italian population with coeliac disease. Europ Rev Med Pharmacolog Sci 2013; 17: 11671173

[12] Malagelada C, De Lorio F, Segui S et al. Functional gut disorders or disordered gut function? Small bowel dysmotility evidenced by an original technique Neurogastroenterol Motility 2012; 24: 223-e105

[13] Rauch S, Krueger K, Turan A et al. Determining small intestinal transit time and pathomorphology in critically ill patients using video capsule technology. Intensive Care Med 2009; 35: 1054-1059

[14] Hellmig S, Von Schöning F, Gadow C et al. Gastric emptying time of fluids and solids in healthy subjects determined by $13 \mathrm{C}$ breath tests: influence of age, sex and body mass index. J Gastroenterol Hepatol 2006; 21: 1832-1838

[15] Niv E, Pinchasovich H, Yanai H. Impact of demographic and clinical parameters on video capsule transit time. Europ J Gastroenterol Hepatol 2016; 28: 1161-1165

[16] Fireman Z, Paz D, Kopelman Y. Capsule endoscopy: Improving transit time and image view. World J Gastroenterol 2005; 11: 5863-5866

[17] Prichard D, Ou G, Galorport C et al. Sham feeding with bacon does not alter transit time or complete examination rate during small bowel capsule endoscopy. Dig Dis Sci 2018; 63: 422-428
[18] Velayos Jimenez B, Fernandez Salazar L, Aller de la Fuente R et al. Study of gastronitestinal transit times with capsule endoscopy. Gastroenterologia y hepatologia 2005; 28: 315-320

[19] Cassilly D, Kantor S, Knight L et al. Gastric emptying of a non-digestible solid: Assessment with simultaneous SmartPill pH and pressure capsule, antroduodenal manometry, gastric emptying scintigraphy. Neurogastroenterol Motil 2008; 20: 311-319

[20] Kalantzis C, Triantafyllou K, Papadopoulos AA et al. Effect of three bowel preparations on video-capsule endoscopy gastric and smallbowel transit time and completeness of the examination. Scand J Gastroenterol 2007; 42: 1120-1126

[21] Camilleri M, Colemont L], Phillips SF et al. Human gastric emptying and colonic filling of solids characterized by a new method. Am J Physiol 1989; 257: G284-290

[22] Bonapace ES, Maurer AH, Davidoff S et al. Whole gut transit scintigraphy in the clinical evaluation of patients with upper and lower gastrointestinal symptoms. Am J Gastroenterol 2000; 95: 2838-2847

[23] Stacher G, Bergmann H. Scintigraphic quantitation of gastrointestinal motor activity and transport: oesophagus and stomach. Eur J Nucl Med 1992; 19: 815-823

[24] Tougas G, Eaker EY, Abell TL et al. Assessment of gastric emptying using a low fat meal: establishment of international control values. Am J Gastroenterol 2000; 95: 1456

[25] Bluck LJ. Recent advances in the interpretation of the (13)C octanoate breath test for gastric emptying. J Breath Res 2009; 3: 034002 